\title{
The Antidiabetic Drug Metformin and Male Reproductive Function: An Overview
}

\author{
Editorial \\ Meneses $\mathrm{MJ}^{1,2}$, Sousa $\mathrm{M}^{2,3}$, Alves $\mathrm{MG}^{1 *}$, Oliveira $\mathrm{PF}^{1,2^{*}}$ \\ ${ }^{1}$ CICS - UBI - Health Sciences Research Centre, University of Beira Interior, Covilhã, Portugal. \\ ${ }^{2}$ Department of Microscopy, Laboratory of Cell Biology and Unit for Multidisciplinary Research in Biomedicine, Abel Salazar Institute of Biomedical \\ Sciences, University of Porto - UMIB/ICBAS/UP, Portugal. \\ ${ }^{3}$ Centre for Reproductive Genetics Professor Alberto Barros, Porto, Portugal.
}

\section{*Corresponding Author:}

Marco G. Alves \& Pedro F. Oliveira,

University of Beira Interior, Av. Infante D. Henrique, 6201-506 Covilhã, Portugal.

Email: alvesmarc@gmail.com \& pfobox@gmail.com

Received: April 15, 2015

Published: April 20, 2015

Citation: Meneses MJ, Sousa M, Alves MG, Oliveira PF (2015) The Antidiabetic Drug Metformin and Male Reproductive Function: An Overview. Int J Diabetol Vasc Dis Res, 3(1e) 1-2. doi: http://dx.doi. org/10.19070/2328-353X-150008e

Copyright: Alves MG (and) Oliveira $\mathbf{P F}^{\mathcal{O}}$ 2015. This is an open-access article distributed under the terms of the Creative Commons Attribution License, which permits unrestricted use, distribution and reproduction in any medium, provided the original author and source are credited.

Diabetes mellitus (DM) is a metabolic disorder characterized by chronic hyperglycemia resulting from defects in insulin action, insulin secretion, or both [1]. These defects lead to disturbances in carbohydrates, lipid and protein metabolism causing systemic complications and co-morbidities, particularly in renal and cardiovascular systems [2]. This disease is reaching pandemic proportions and a recent report estimated that more than 300 million of people worldwide already have DM and, alarmingly, some projections suggest that the number of diabetic patients will reach nearly 600 million by the year 2035 [3].

In contrast with DM prevalence, the fertility rates have been declining in the recent decades. Interestingly, these two events appear to be related for various reasons [4]. One of these reasons is the alarming increase of the number of men developing DM during the reproductive age. The vast majority of type $1 \mathrm{DM}$ cases are diagnosed before the age of 30 [5] and there is an increasing number of children and adolescents with type $2 \mathrm{DM}$ (T2DM) [6]. Thus, due to its complexity and the dramatic increase of diabetic patients, it urges to study the implications of DM in human reproductive health, particularly on male fertility.

When prescribing a treatment for DM, a complex analysis is needed to find the most suitable antidiabetic drug [7]. Several of these compounds, if not all, have the ability to modulate cellular metabolism in a manner that may benefit some organs, but damage others. Metformin is a biguanide, considered the first-line treatment for T2DM [7]. It is an insulin-sensitizing drug that exerts its anti-hyperglycemic effects by increasing the skeletal muscle uptake of glucose and reducing the absorption of glucose in the intestinal mucosa $[8,9]$. Moreover, it blocks liver gluconeogenesis through regulation of the gluconeogenic flux [9]. Nevertheless, although a common feature has already been attributed to this drug in promoting the activity of AMP-activated protein kinase (AMPK) [10], the exact molecular mechanisms of metformin action remain to be fully disclosed, despite several decades of research focused on this drug action [9].

While metformin is presently being used for the management of female reproductive function, namely for the treatment of polycystic ovary syndrome [8], its effects on human male reproductive function remain largely unknown. The vast majority of the data available was obtained in studies conducted on animal models, particularly rodents. Moreover, the existing results remain controversial with some conflicting data. While data derived from exposing healthy animals to metformin resulted in the observation of deleterious reproductive effects, data obtained from diabetic animal models reinforce the therapeutic potential of this drug in protecting male reproductive health against the deleterious effects of DM. In fact, healthy male animals exposed to metformin exhibited adverse reproductive outcomes that involved decreased testosterone production [11], reduced testes size and seminiferous tubules diameter, with reduction on Sertoli cell numbers and concomitant decrease in sperm quality parameters [12]. Contrastingly, in studies where diabetic animal were exposed to metformin, it has been consistently reported that this drug can improve the male reproductive function and the spermatogenic index $[13,14]$. Indeed, metformin administration was able to restore testosterone, LH and FSH levels on the testes of streptozotocin-induced diabetic rats [13]. A protective role has also been ascribed for metformin on male reproductive function of diabetic animals, not only by improving steroidogenesis and the antioxidant status of the testes (usually linked to an overproduction of ROS due to the hyperglycemic environment), but also by leading to an amelioration of sperm concentration and motility and of the percentage of morphologically normal sperm [13-16]. Although the literature shows some contradictory results when examining the effect of DM on human and animal sperm parameters and sperm quality markers, most of the studies reported a positive influence of metformin on sperm parameters $[17,18]$. In fact, men with metabolic syndrome treated with metformin presented a significantly amelioration on sperm concentration, motility and morphology, which was attributed to the increase of $\mathrm{LH}$ and testosterone lev- 
els [18]. Furthermore, male diabetics present an increased risk of developing particular sexual disorders, such as erectile dysfunction or retrograde ejaculation [19]. The treatment with metformin was shown to increase the contractility in the corpora cavernosa, improving the erectile function observed in a hypertensive model. The exposure to this drug was also reported to increase endothelial nitric oxide synthase phosphorylation, an important factor in erectile function, which is normally decreased in patients with erectile dysfunction, namely male diabetic individuals [20].

In vitro studies also support the therapeutic potential of metformin in protecting male reproductive health. In a recent study, our team has reported a stimulation of lactate production in rat Sertoli cells exposed to metformin [8]. Lactate production by Sertoli cells is a crucial event since this metabolite is the preferred energy substrate for developing germ cells and has an important anti-apoptotic effect [21]. Thus, the increase in lactate production caused by metformin may be positive for spermatogenesis and male reproductive function. Several other studies provided evidence that metformin acts as an inhibitor of complex I of the mitochondrial electron transport chain $[22,23]$, which leads to a decrease in oxidative metabolism and, consequently, to an increase in anaerobic respiration and lactate secretion [23].

Metformin has also been reported to be valuable as a protective compound in the supplementation of sperm cryopreservation media [24]. Cryopreservation is a method routinely used to conserve male spermatozoa for, among others, bypassing infertility issues [25]. However, this technique may cause permanent damage to spermatozoa such as loss of motility, reduced DNA integrity, and apoptosis [26]. Not only exposure of fresh semen to metformin did not induce negative effects on spermatozoa quality, as the addition of this drug to the cryopreservation protocol led to an improvement in the fertilization rates and a reduction in the percentage of abnormal zygotes after in vitro fertilization [24]. Thus, metformin is being regarded as a potential additive to cryopreservation media since it presents beneficial effects in improving the quality of frozen semen.

In sum, although data from studies involving human individuals or human material are scarce, it is generally accepted that metformin improves several aspects in the reproductive function of diabetic individuals. Metformin is one of the most prescribed anti diabetic drugs, but its effects on male fertility have been lightly investigated. It is imperative to fully scrutinize the molecular mechanisms behind the effects of metformin on the overall human male reproductive function, and those that may individually affect testicular cells, spermatogenesis, sperm production and sperm maturation. Definitely, as fertility rates dramatically decrease, the relevance of studying the influence of drugs that protect the male reproductive function exponentially increases.

\section{References}

[1]. American Diabetes Association. (2014) Diagnosis and classification of diabetes mellitus. Diabetes Care 37(Supplement 1): S81-S90.

[2]. Alves MG, Martins AD, Rato L, Moreira PI, Socorro S, et al. (2013) Molecular mechanisms beyond glucose transport in diabetes-related male infertility. Biochimica et Biophysica Acta (BBA)-Molecular Basis of Disease 1832(5): 626-635.

[3]. Guariguata L, Whiting D, Hambleton I, Beagley J, Linnenkamp U, et al. (2014) Global estimates of diabetes prevalence for 2013 and projections for 2035. Diabetes research and clinical practice 103(2): 137-149.

[4]. Vignera S, Condorelli R, Vicari E, D'agata R, Calogero AE (2012) Diabetes mellitus and sperm parameters. Journal of andrology 33(2): 145-153.
[5]. Patterson CC, Dahlquist GG, Gyürüs E, Green A, Soltész G, et al. (2009) Incidence trends for childhood type 1 diabetes in Europe during 1989-2003 and predicted new cases 2005-20: a multicentre prospective registration study. The Lancet 373(9680): 2027-2033.

[6]. Chen L, Magliano DJ, Zimmet PZ (2012) The worldwide epidemiology of type 2 diabetes mellitus - present and future perspectives. Nature Reviews Endocrinology 8(4): 228-236.

[7]. Qaseem A, Humphrey LL, Sweet DE, Starkey M, Shekelle P (2012) Oral pharmacologic treatment of type 2 diabetes mellitus: a clinical practice guideline from the American College of Physicians. Annals of internal medicine 156(3): 218-231.

[8]. Alves MG, Martins AD, Vaz CV, Correia S, Moreira PI, et al. (2014) Metformin and male reproduction: effects on Sertoli cell metabolism. British journal of pharmacology 171(4): 1033-1042.

[9]. Viollet B, Guigas B, Garcia NS, Leclerc J, Foretz M, et al. (2012) Cellular and molecular mechanisms of metformin: an overview. Clinical science 122(6): 253-270.

[10]. Zhou G, Myers R, Li Y, Chen Y, Shen X, et al. (2001) Role of AMP-activated protein kinase in mechanism of metformin action. Journal of clinical investigation 108(8): 1167.

[11]. Tartarin P, Moison D, Guibert E, Dupont J, Habert R, et al. (2012) Metformin exposure affects human and mouse fetal testicular cells. Human Reproduction 27(11): 3304-14.

[12]. Adaramoye O, Akanni O, Adesanoye O, Labo-Popoola O, Olaremi O (2012) Evaluation of toxic effects of metformin hydrochloride and glibenclamide on some organs of male rats. Nigerian journal of physiological sciences 27(2): 137-44.

[13]. Nasrolahi O, Khaneshi F, Rahmani F, Razi M (2013) Honey and metformin ameliorated diabetes-induced damages in testes of rat; correlation with hormonal changes. Iranian journal of reproductive medicine 11(12): 1013-20.

[14]. Fang X, Xu QY, Jia C, Peng YF (2012) Metformin improves epididymal sperm quality and antioxidant function of the testis in diet-induced obesity rats. Zhonghua Nan Ke Xue 18(2): 146-9.

[15]. Attia SM, Helal GK, Alhaider AA (2009) Assessment of genomic instability in normal and diabetic rats treated with metformin. Chemico-Biological Interactions 180(2): 296-304.

[16]. Rabbani SI, Devi K, Khanam S (2010) Role of Pioglitazone with Metformin or Glimepiride on Oxidative Stress-induced Nuclear Damage and Reproductive Toxicity in Diabetic Rats. Malaysian Journal of Medical Sciences 17(1): 3-11.

[17]. Attia SM, Helal GK, Alhaider AA (2009) Assessment of genomic instability in normal and diabetic rats treated with metformin. Chemico-biological interactions 180(2): 296-304.

[18]. Morgante G, Tosti C, Orvieto R, Musacchio MC, Piomboni P, et al. (2011) Metformin improves semen characteristics of oligo-terato-asthenozoospermic men with metabolic syndrome. Fertility and sterility 95(6): 2150-2152.

[19]. Phé V, Rouprêt M (2012) Erectile dysfunction and diabetes: a review of the current evidence-based medicine and a synthesis of the main available therapies. Diabetes \& metabolism 38(1): 1-13.

[20]. Labazi H, Wynne BM, Tostes R, Webb RC (2013) Metformin treatment improves erectile function in an angiotensin II model of erectile dysfunction. The journal of sexual medicine 10(9): 2154-2164.

[21]. Dias TR, Martins AD, Reis VP, Socorro S, Silva BM, et al. (2013) Glucose Transport and Metabolism in Sertoli Cell: Relevance for Male Fertility. Current Chemical Biology 7(3): 282-293.

[22]. Andrzejewski S, Gravel SP, Pollak M, St-Pierre J (2014) Metformin directly acts on mitochondria to alter cellular bioenergetics. Cancer \& Metabolism 2: 12 .

[23]. Owen M, Doran E, Halestrap A (2000) Evidence that metformin exerts its anti-diabetic effects through inhibition of complex 1 of the mitochondrial respiratory chain. Biochemical Journal 348(3): 607-614.

[24]. Bertoldo MJ, Guibert E, Tartarin P, Guillory V, Froment P (2014) Effect of metformin on the fertilizing ability of mouse spermatozoa. Cryobiology 68(2): 262-268.

[25]. Dias TR, Alves MG, Tomas GD, Socorro S, Silva BM, et al. (2014) White tea as a promising antioxidant medium additive for sperm storage at room temperature: a comparative study with green tea. Journal of agricultural and food chemistry 62(3): 608-617.

[26]. Boitrelle F, Albert M, Theillac C, Ferfouri F, Bergere M, et al. (2012) Cryopreservation of human spermatozoa decreases the number of motile normal spermatozoa, induces nuclear vacuolization and chromatin decondensation. Journal of andrology 33(6): 1371-1378. 